\title{
Bias-Corrected Maximum Likelihood Estimators of the Parameters of the Unit-Weibull Distribution
}

\author{
J. Mazucheli \\ Universidade Estadual \\ de Maringá
}

\author{
A. F. B. Menezes \\ Universidade Estadual \\ de Maringá
}

\author{
F. Alqallaf \\ Kuwait University
}

\author{
M. E. Ghitany \\ Kuwait University
}

\begin{abstract}
It is well known that the maximum likelihood estimates (MLEs) have appealing statistical properties. Under fairly mild conditions their asymptotic distribution is normal, and no other estimator has a smaller asymptotic variance. However, in finite samples the maximum likelihood estimates are often biased estimates and the bias disappears as the sample size grows. Mazucheli, Menezes, and Ghitany (2018b) introduced a two-parameter unit-Weibull distribution which is useful for modeling data on the unit interval, however its MLEs are biased in finite samples. In this paper, we adopt three approaches for bias reduction of the MLEs of the parameters of unit-Weibull distribution. The first approach is the analytical methodology suggested by Cox and Snell (1968), the second is based on parametric bootstrap resampling method, and the third is the preventive approach introduced by Firth (1993). The results from Monte Carlo simulations revealed that the biases of the estimates should not be ignored and the bias reduction approaches are equally efficient. However, the first approach is easier to implement. Finally, applications to two real data sets are presented for illustrative purposes.
\end{abstract}

Keywords: unit-Weibull distribution, bias-correction, bootstrap, Monte Carlo simulation.

\section{Introduction}

Recently, Mazucheli et al. (2018b) proposed a new two-parameter probability distribution for data analysis on the unit interval $(0,1)$. This new proposal arose from a transformation of a two-parameter Weibull random variable and was named the unit-Weibull (UW) distribution. Specifically, the UW distribution was obtained by applying the transformation $X=\mathrm{e}^{-Y}$, where $Y$ follows a Weibull distribution with scale parameter $\alpha$ and shape parameter $\beta$. The authors discussed important properties of UW distribution and in two applications showed that it outperforms 8 well-known competing distributions on the unit interval, including the Beta and Kumaraswamy distributions. More recently, Mazucheli, Menezes, Fernandes, de Oliveira, and Ghitany (2019b) introduced an alternative quantile regression model by using the unit-Weibull distribution. The proposed model has proved to be very useful in real applications.

The probability density function (p.d.f.) and cumulative distribution function (c.d.f.) of the 
UW distribution, respectively, are expressed as

$$
f(x \mid \alpha, \beta)=\frac{1}{x} \alpha \beta(-\log x)^{\beta-1} \exp \left[-\alpha(-\log x)^{\beta}\right]
$$

and

$$
F(x \mid \alpha, \beta)=\exp \left[-\alpha(-\log x)^{\beta}\right]
$$

where $0<x<1$ and $\alpha, \beta>0$. Note that $\alpha$ is no longer a scale parameter, since $f(\alpha x \mid$ $\alpha, \beta) \neq \frac{1}{\alpha} f(x \mid 1, \beta)$. Mazucheli et al. (2018b) showed that UW distribution includes three special cases: the standard uniform distribution $(\alpha=\beta=1)$, the power function distribution $(\beta=1)$ and the unit-Rayleigh distribution $(\beta=2)$.

Figure 1 shows some possible shapes of the p.d.f. for selected values of the parameters $\alpha$ and $\beta$. Note that the p.d.f. may assume different forms according to the values of its parameters: symmetrical unimodal; asymmetric unimodal on the left; asymmetric unimodal on the right; bathtub shape; increasing exponential; decreasing and constant exponential. This flexibility in its shape makes UW distribution a flexible alternative for analyzing data on the unit interval.
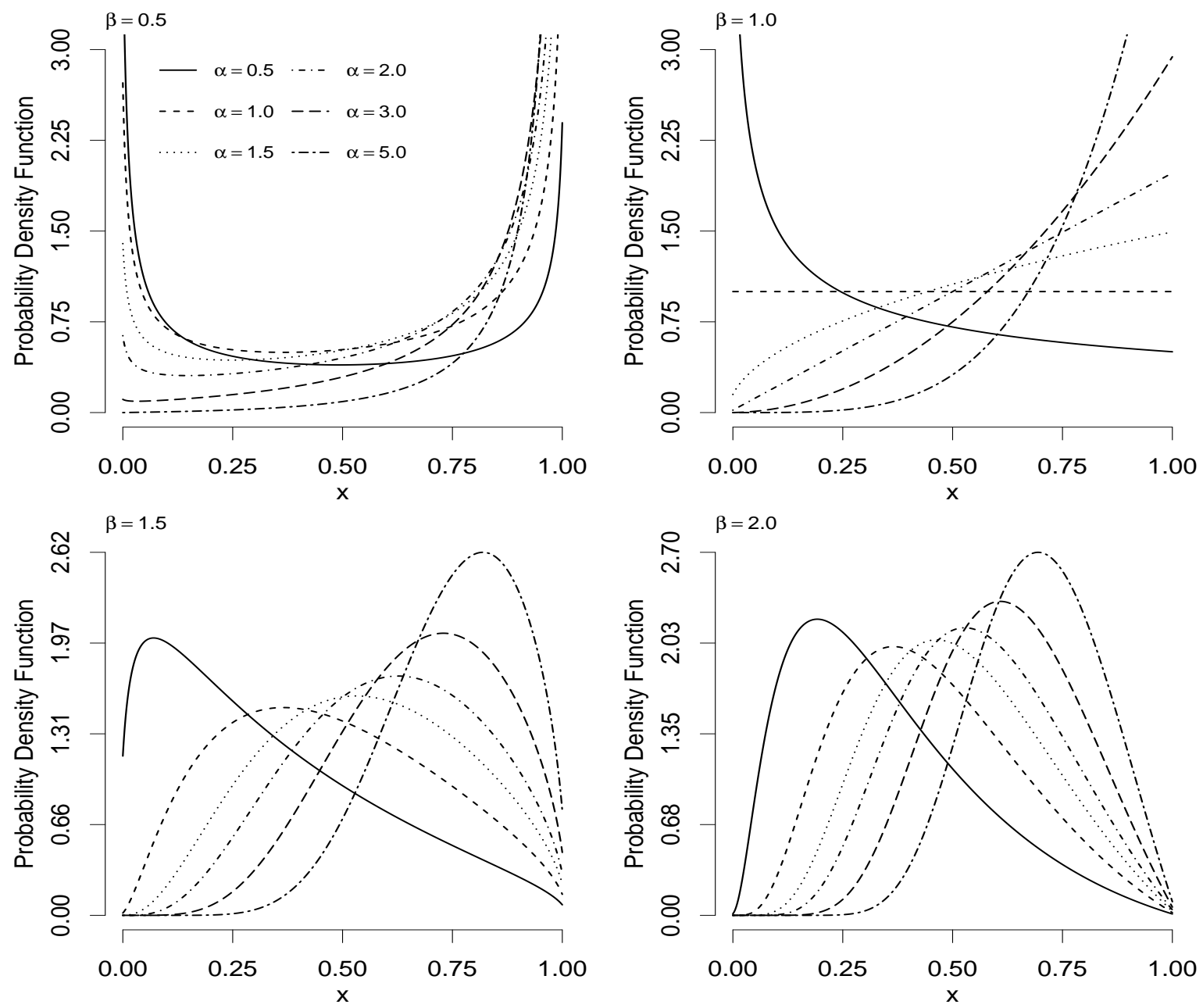

Figure 1: Probability density function of the UW distribution for selected values of $\alpha$ and $\beta$

From the point of view of parametric inference, the parameters estimation is the utmost importance for any probability distribution under consideration. Among all estimation methods, surely the maximum likelihood method (Pawitan 2001; Millar 2011) enjoys great popularity in practice because (i) its underlying motivation is simple and intuitive, (ii) it has several attractive properties, for instance they are asymptomatically unbiased, consistent and asymptotically normally distributed (Edwards 1992; Lehmann 1999) and (iii) it is possible to 
measure the accuracy of point estimation. The majority of these properties are not shared with other parameter estimation methods.

Nonetheless, the most cited properties of the maximum likelihood method essentially rely on the large sample size condition. In particular, the maximum likelihood estimators have biases of order $\mathcal{O}\left(n^{-1}\right)$, which are commonly ignored in practice. As demonstrated by Mazucheli et al. (2018b) the maximum likelihood estimators of the parameters associated to the UW distribution are positively biased in small sample $(n=10,20,30,40$ and 50), this means that the expected value of estimators exceeds the true value of parameters, that is, the MLE $\widehat{\alpha}$ and $\widehat{\beta}$ overestimate $\alpha$ and $\beta$, respectively. Taking this into account, it is important to develop nearly unbiased estimators for the UW distribution, as considered for the Beta distribution in Cribari-Neto and Vasconcellos (2002).

In this paper, we shall adopt three corrective approaches to derive modified MLEs that are unbiased to second order: (i) the bias-correction method introduced by Cox and Snell (1968), with analytical expressions for the biases, (ii) the parametric bootstrap resampling method by (Efron 1982) and (iii) the preventive approach introduced by Firth (1993).

As can be seen in our simulation study, the proposed three corrected MLEs are extremely accurate for small and moderate sample sizes. Furthermore, they are far superior than the uncorrected MLEs with respect to consistency and efficiency. Additionally, the first two methods have closed-form expressions, which means they are quite attractive and easy to compute. Bias-corrections of the MLEs of the parameters of many distributions have been applied successfully in the literature. Interested readers may refer to Cribari-Neto and Vasconcellos (2002), Saha and Paul (2005), Lemonte, Cribari-Neto, and Vasconcellos (2007), Giles (2012), Schwartz, Godwin, and Giles (2013), Giles, Feng, and Godwin (2013), Ling and Giles (2014), Schwartz and Giles (2016), Wang and Wang (2017), Mazucheli, Menezes, and Dey (2018a), Mazucheli, Menezes, and Nadarajah (2017), Mazucheli and Dey (2018), Mazucheli, Menezes, and Dey (2019a), Menezes and Mazucheli (2018), Reath, Dong, and Wang (2018) and the references therein.

The rest of the paper is organized as follows. In Sections 2 and 3 we described the maximum likelihood estimators and derived their bias-corrected estimators for the parameters of the UW distribution. The performance of the bias adjusted estimators are investigated by Monte Carlo simulation in Section 4. Real applications in Section 5 are given for illustrative purposes. Finally, some remarks in Section 6 closes the paper.

\section{Maximum likelihood estimation}

Let $\mathbf{x}=\left(x_{1}, \ldots, x_{n}\right)$ be a random sample of size $n$ from the UW distribution with p.d.f. (1). Then, the log-likelihood function of $\boldsymbol{\theta}=\left(\theta_{1}, \theta_{2}\right)=(\alpha, \beta)$ is given by

$\ell=\ell(\boldsymbol{\theta} \mid \mathbf{x})=n(\log \alpha+\log \beta)-\sum_{i=1}^{n} \log x_{i}+(\beta-1) \sum_{i=1}^{n} \log \left(-\log x_{i}\right)-\alpha \sum_{i=1}^{n}\left(-\log x_{i}\right)^{\beta}$.

The maximum likelihood estimate $\widehat{\boldsymbol{\theta}}$ of $\boldsymbol{\theta}$ is obtained by solving the non-linear equations

$$
\begin{gathered}
\frac{\partial \ell}{\partial \alpha}=\frac{n}{\alpha}-\sum_{i=1}^{n}\left(-\log x_{i}\right)^{\beta}=0 \\
\frac{\partial \ell}{\partial \beta}=\frac{n}{\beta}+\sum_{i=1}^{n} \log \left(-\log x_{i}\right)-\alpha \sum_{i=1}^{n}\left(-\log x_{i}\right)^{\beta} \log \left(-\log x_{i}\right)=0 .
\end{gathered}
$$

The equation (4) can be solved algebraically for $\alpha$, giving $\widehat{\alpha}(\beta)=\frac{n}{\sum_{i=1}^{n}\left(-\log x_{i}\right)^{\beta}}$. 
To obtain $\widehat{\beta}$, we substitute $\widehat{\alpha}(\beta)$ into (5) and solve for $\beta$. We have

$$
g(\beta)=\frac{n}{\beta}+\sum_{i=1}^{n} \log \left(-\log x_{i}\right)-\frac{n \sum_{i=1}^{n}\left(-\log x_{i}\right)^{\beta} \log \left(-\log x_{i}\right)}{\sum_{i=1}^{n}\left(-\log x_{i}\right)^{\beta}}=0 .
$$

Mazucheli et al. (2018b) proposed a starting-point strategy using the fact that UW cumulative distribution function can be linearized. As a result, one can obtain least squares estimates of $\alpha$ and $\beta$ by fitting a linear regression line, which can be used as initial guess to solve numerically equation (6).

From Mazucheli et al. (2018b), the expected Fisher information matrix is given by

$$
\mathbf{I}(\boldsymbol{\theta})=\left[\mathbf{I}_{i j}(\boldsymbol{\theta})\right]=\mathbb{E}\left[-\frac{\partial^{2}}{\partial \theta_{i} \partial \theta_{j}} \ell(\boldsymbol{\theta} \mid \mathbf{x})\right], \quad i, j=1,2,
$$

where

$$
\begin{aligned}
\mathbf{I}_{11}(\boldsymbol{\theta}) & =\frac{n}{\alpha^{2}}, \\
\mathbf{I}_{12}(\boldsymbol{\theta}) & =\mathbf{I}_{21}(\boldsymbol{\theta})=\frac{n}{\alpha \beta}[1-\gamma-\log (\alpha)], \\
\mathbf{I}_{22}(\boldsymbol{\theta}) & =\frac{n}{6 \beta^{2}}\left[6(1-\gamma-\log \alpha)^{2}+\pi^{2}\right],
\end{aligned}
$$

where $\gamma \approx 0.577215$ is the Euler's constant. Its inverse, $\mathbf{I}^{-1}(\boldsymbol{\theta})=\left[\mathbf{I}^{i j}(\boldsymbol{\theta})\right], i, j=1,2$, has the elements

$$
\begin{aligned}
\mathbf{I}^{11}(\boldsymbol{\theta}) & =\frac{\alpha^{2}}{n \pi^{2}}\left[6(1-\gamma-\log \alpha)^{2}+\pi^{2}\right], \\
\mathbf{I}^{12}(\boldsymbol{\theta}) & =\mathbf{I}^{21}(\boldsymbol{\theta})=-\frac{6 \alpha \beta}{n \pi^{2}}(1-\gamma-\log \alpha), \\
\mathbf{I}^{22}(\boldsymbol{\theta}) & =\frac{6 \beta^{2}}{n \pi^{2}}
\end{aligned}
$$

Note that $\mathbf{I}^{-1}(\widehat{\boldsymbol{\theta}})$ is the estimated variance-covariance matrix of the MLE $\widehat{\boldsymbol{\theta}}$ of $\boldsymbol{\theta}$.

\section{Bias-corrected maximum likelihood estimators}

Maximum likelihood estimators are usually biased, typically they have biases of order $\mathcal{O}\left(n^{-1}\right)$. For this reason, we use the following three methods to reduce the bias of the MLEs.

(i) Cox-Snell method

For a $p$-dimensional parameter vector $\boldsymbol{\theta}=\left(\theta_{1}, \theta_{2}, \ldots, \theta_{p}\right)$, Cox and Snell (1968) showed that when the sample data are independent, but not necessarily identically distributed, the bias of the $r$-th MLE $\widehat{\theta}_{r}$ of $\theta_{r}$, is obtained as

$$
\operatorname{Bias}\left(\widehat{\theta}_{r}\right)=\sum_{i=1}^{p} \sum_{j=1}^{p} \sum_{k=1}^{p} \mathbf{I}^{r i}(\boldsymbol{\theta}) \mathbf{I}^{j k}(\boldsymbol{\theta})\left[0.5 \mathbf{I}_{i j k}(\boldsymbol{\theta})+\mathbf{I}_{i j, k}(\boldsymbol{\theta})\right]+\mathcal{O}\left(n^{-2}\right), \quad r=1, \ldots, p,
$$

where $\mathbf{I}^{i j}(\boldsymbol{\theta})$ is the $(i, j)$-th element of the inverse expected information matrix $\mathbf{I}^{-1}(\boldsymbol{\theta})$,

$$
\mathbf{I}_{i j k}(\boldsymbol{\theta})=\mathbb{E}\left[\frac{\partial^{3}}{\partial \boldsymbol{\theta}_{i} \partial \boldsymbol{\theta}_{j} \partial \boldsymbol{\theta}_{k}} \ell(\boldsymbol{\theta} \mid \mathbf{x})\right]
$$


and

$$
\mathbf{I}_{i j, k}(\boldsymbol{\theta})=\mathbb{E}\left[\frac{\partial^{2}}{\partial \boldsymbol{\theta}_{i} \partial \boldsymbol{\theta}_{j}} \ell(\boldsymbol{\theta} \mid \mathbf{x}) \frac{\partial}{\partial \boldsymbol{\theta}_{k}} \ell(\boldsymbol{\theta} \mid \mathbf{x})\right]
$$

Therefore, the bias corrected estimator (BCE) $\widehat{\theta}_{r(B C E)}$ of the MLE $\widehat{\theta}_{r}$ is given by

$$
\widehat{\theta}_{r(B C E)}=\widehat{\theta_{r}}-\widehat{\operatorname{Bias}}\left(\widehat{\theta}_{r}\right), \quad r=1, \ldots, p .
$$

where $\widehat{\operatorname{Bias}}\left(\widehat{\theta}_{r}\right)$ is the bias of $\widehat{\theta}_{r}$ evaluated at the MLE $\widehat{\boldsymbol{\theta}}$. Clearly, we would expect that the BCEs will have better sampling properties than the uncorrected MLEs. For example, it is expected that the standard errors of BCEs to be smaller than that of the MLEs.

For the UW distribution, we have

$$
\begin{aligned}
\mathbf{I}_{111}(\boldsymbol{\theta}) & =\frac{2 n}{\alpha^{3}}, \\
\mathbf{I}_{112}(\boldsymbol{\theta})= & \mathbf{I}_{121}(\boldsymbol{\theta})=\mathbf{I}_{211}(\boldsymbol{\theta})=0 \\
\mathbf{I}_{122}(\boldsymbol{\theta})= & \mathbf{I}_{212}(\boldsymbol{\theta})=\mathbf{I}_{221}(\boldsymbol{\theta})=-\frac{n}{\alpha \beta^{2}}\left[(1-\gamma-\log \alpha)^{2}+\frac{\pi^{2}}{6}-1\right], \\
\mathbf{I}_{222}(\boldsymbol{\theta})= & \frac{n}{\beta^{3}}\left[(\log \alpha)^{3}+3(\gamma-1)(\log \alpha)^{2}+\left(3 \gamma^{2}-6 \gamma+\frac{\pi^{2}}{2}\right) \log (\alpha)\right. \\
& \left.\quad+\gamma^{3}-3 \gamma^{2}+\frac{\pi^{2}}{2} \gamma-\frac{\pi^{2}}{2}+2 \zeta(3)+2\right] .
\end{aligned}
$$

where $\zeta(s)=\sum_{n=1}^{\infty} \frac{1}{n^{s}}$ is the Riemann's Zeta function.

Also, we have

$$
\begin{aligned}
& \mathbf{I}_{11,1}(\boldsymbol{\theta})=\mathbf{I}_{11,2}=0, \\
& \mathbf{I}_{12,1}(\boldsymbol{\theta})=\mathbf{I}_{21,1}(\boldsymbol{\theta})=-\frac{n}{\alpha^{2} \beta}[\log (\alpha)+\gamma-2], \\
& \mathbf{I}_{12,2}(\boldsymbol{\theta})= \mathbf{I}_{21,2}(\boldsymbol{\theta})=\frac{n}{\alpha \beta^{2}}\left[(\log \alpha)^{2}+(2 \gamma-3) \log (\alpha)+\gamma^{2}-3 \gamma+\frac{\pi^{2}}{6}+1\right], \\
& \mathbf{I}_{22,1}(\boldsymbol{\theta})= \frac{n}{\alpha \beta^{2}}\left[(\log \alpha)^{2}+2(\gamma-2) \log (\alpha)+\gamma^{2}-4 \gamma+\frac{\pi^{2}}{6}+2\right], \\
& \mathbf{I}_{22,2}(\boldsymbol{\theta})=-\frac{n}{\beta^{3}}\left[(\log \alpha)^{3}+(3 \gamma-5)(\log \alpha)^{2}+\left(3 \gamma^{2}-10 \gamma+\frac{\pi^{2}}{2}+4\right) \log (\alpha)\right. \\
&\left.\quad+\gamma^{3}-5 \gamma^{2}+\left(\frac{\pi^{2}}{2}+4\right) \gamma-\frac{5}{6} \pi^{2}+2 \zeta(3)\right] .
\end{aligned}
$$

By replacing these terms in Equation (7), we obtain the second order biases of $\widehat{\alpha}$ and $\widehat{\beta}$, respectively, as follows

$$
\begin{aligned}
\operatorname{Bias}(\widehat{\alpha}) & =\frac{0.303963548}{n} \alpha[\log (\alpha)+2.948557322][\log (\alpha)+0.7443481081] \\
\operatorname{Bias}(\widehat{\beta}) & =-\frac{0.11 \times 10^{-8}}{n} \beta[\log (\alpha)+35414.40358][\log (\alpha)-35412.67631]
\end{aligned}
$$

Note that $\widehat{\operatorname{Bias}}(\widehat{\alpha})$ depends only on the MLE $\widehat{\alpha}$ while $\widehat{\operatorname{Bias}}(\widehat{\beta})$ depends on both the MLEs $\widehat{\alpha}$ and $\widehat{\beta}$. It is important to mention that the validity of the expressions (9) and (10) can be verified numerically through the the coxsnell.bc function, available in the mle.tools library (Mazucheli et al. 2017) of the R environment of the R environment (R Core Team 2017). 
(ii) Parametric bootstrap method

A different approach to derive second-order bias-corrected estimators is based on the parametric bootstrap resampling scheme (Efron 1982; Davison and Hinkley 1997). The method is as follows. Let $X_{1}, X_{2}, \ldots, X_{n}$ be a random sample from a distribtion with p.d.f. $f(x ; \boldsymbol{\theta})$, where $\boldsymbol{\theta}=\left(\theta_{1}, \theta_{2}, \ldots, \theta_{p}\right)$. Based on this random sample, we compute the MLE $\widehat{\boldsymbol{\theta}}$ of $\boldsymbol{\theta}$. Now, we generate $B$ independent random samples, called parametric bootstrap samples, from the distribution with p.d.f. $f(x ; \widehat{\boldsymbol{\theta}})$. The estimated bias of the MLE $\widehat{\theta_{r}}$ is given by

$$
\widehat{\operatorname{Bias}}\left(\widehat{\theta}_{r}\right)=\frac{1}{B} \sum_{j=1}^{B} \widehat{\theta}_{r(j)}-\widehat{\theta}_{r}, \quad r=1, \ldots, p
$$

where $\widehat{\theta}_{r(j)}$ is the MLE of $\theta_{r}$ obtained from the $j$-th bootstrap sample. Therefore, the parametric bootstrap estimator (PBE) $\widehat{\theta}_{r(P B E)}$ of the MLE of $\widehat{\theta}_{r}$ is given by

$$
\widehat{\theta}_{r(P B E)}=2 \widehat{\theta}_{r}-\frac{1}{B} \sum_{j=1}^{B} \widehat{\theta}_{r(j)}, \quad r=1, \ldots, p .
$$

The parametric bootstrap method is described in detail by Efron and Tibshirani (1993).

(iii) Firth method

An alternative approach to obtain bias-corrected estimators proposed by Firth (1993) consists of transforming the score vector $\mathbf{U}(\boldsymbol{\theta})=\frac{\partial \ell}{\partial \boldsymbol{\theta}}$ before obtaining the maximum likelihood estimators. This method is known as the preventive method. The modified score vector is defined by

$$
\mathbf{U}^{*}(\boldsymbol{\theta})=\mathbf{U}(\boldsymbol{\theta})-\mathbf{I}(\boldsymbol{\theta}) \mathcal{B}(\widehat{\boldsymbol{\theta}})
$$

where $\mathbf{I}(\boldsymbol{\theta})$ is the expected information matrix and $\mathcal{B}(\widehat{\boldsymbol{\theta}})$ is the second-order bias vector with the components defined in (9) and (10).

\section{Simulation study}

The aim of the following simulation study is to evaluate the performance of the various estimators (MLEs, BCEs, PBEs and FBEs) of the parameters of the UW distribution. To do so, we generated data from the UW distribution by taking $X=\mathrm{e}^{-Y}$, where $Y \sim \operatorname{Weibull}(\alpha, \beta)$. The generated samples are of sizes $n=10,20,30,40,50$, from the UW distribution with parameters $\alpha, \beta=0.5,1.0,1.5,2.0$. The number of Monte Carlo samples is $M=10,000$ and the number of bootstrap replications is $B=1,000$. The Monte Carlo study is performed in Ox Console (Doornik 2007), the MaxBFGS function was used to obtain the maximum likelihood estimates and the SolveNLE to obtain the Firth bias corrected estimates.

To evaluate the accuracy of the parameter estimates, the bias and root mean-squared error of the estimates are reported in Tables 1-4. From these it is possible to conclude that the BCEs, PBEs and FBEs have smaller absolute biases and smaller root mean-squared errors than those of the MLEs, particularly for small and moderate sample sizes. 
Table 1: Estimated bias (root mean-squared error) for $\alpha$ and $\beta,(\beta=0.5)$

\begin{tabular}{|c|c|c|c|c|c|c|c|c|c|}
\hline \multirow[b]{2}{*}{$\alpha$} & \multirow[b]{2}{*}{$n$} & \multicolumn{4}{|c|}{ Estimator of $\alpha$} & \multicolumn{4}{|c|}{ Estimator of $\beta$} \\
\hline & & MLE & $\mathrm{BCE}$ & PBE & FBE & MLE & $\mathrm{BCE}$ & PBE & FBE \\
\hline \multirow{5}{*}{0.5} & 10 & $0.015(0.246)$ & $0.005(0.219)$ & $-0.007(0.208)$ & $0.017(0.211)$ & $0.083(0.191)$ & $0.003(0.148)$ & $-0.015(0.145)$ & $-0.026(0.134)$ \\
\hline & 20 & $0.004(0.153)$ & $0.002(0.147)$ & $-0.000(0.146)$ & $0.005(0.146)$ & $0.037(0.108)$ & $-0.000(0.094)$ & $-0.004(0.094)$ & $-0.006(0.093)$ \\
\hline & 30 & $0.003(0.123)$ & $0.001(0.120)$ & $0.000(0.120)$ & $0.002(0.120)$ & $0.024(0.082)$ & $-0.000(0.075)$ & $-0.002(0.075)$ & $-0.003(0.074)$ \\
\hline & 40 & $0.002(0.106)$ & $0.001(0.104)$ & $0.000(0.104)$ & $0.002(0.104)$ & $0.018(0.069)$ & $-0.000(0.064)$ & $-0.001(0.064)$ & $-0.002(0.064)$ \\
\hline & 50 & $0.001(0.095)$ & $0.000(0.093)$ & $0.000(0.093)$ & $0.001(0.093)$ & $0.014(0.060)$ & $0.000(0.057)$ & $-0.000(0.057)$ & $-0.001(0.057)$ \\
\hline \multirow{5}{*}{1.0} & 10 & $0.102(0.501)$ & $-0.001(0.363)$ & $-0.050(0.368)$ & $-0.001(0.364)$ & $0.086(0.200)$ & $0.005(0.156)$ & $-0.012(0.151)$ & $-0.015(0.146)$ \\
\hline & 20 & $0.039(0.272)$ & $-0.001(0.242)$ & $-0.011(0.236)$ & $0.000(0.244)$ & $0.039(0.111)$ & $0.002(0.097)$ & $-0.002(0.097)$ & $-0.005(0.094)$ \\
\hline & 30 & $0.025(0.208)$ & $0.000(0.194)$ & $-0.004(0.192)$ & $0.001(0.198)$ & $0.025(0.084)$ & $0.001(0.076)$ & $-0.001(0.076)$ & $-0.002(0.075)$ \\
\hline & 40 & $0.017(0.178)$ & $-0.001(0.168)$ & $-0.003(0.168)$ & $0.001(0.170)$ & $0.019(0.071)$ & $0.001(0.066)$ & $0.001(0.065)$ & $-0.002(0.064)$ \\
\hline & 50 & $0.013(0.156)$ & $-0.001(0.150)$ & $-0.002(0.149)$ & $0.000(0.152)$ & $0.015(0.062)$ & $0.001(0.058)$ & $0.001(0.058)$ & $-0.002(0.056)$ \\
\hline \multirow{5}{*}{1.5} & 10 & $0.284(0.897)$ & $-0.005(0.533)$ & $-0.070(0.688)$ & $-0.024(0.540)$ & $0.085(0.192)$ & $0.004(0.148)$ & $-0.009(0.150)$ & $-0.016(0.146)$ \\
\hline & 20 & $0.110(0.454)$ & $0.002(0.374)$ & $-0.023(0.365)$ & $-0.002(0.368)$ & $0.039(0.109)$ & $0.001(0.095)$ & $-0.002(0.094)$ & $-0.004(0.096)$ \\
\hline & 30 & $0.069(0.329)$ & $0.003(0.292)$ & $-0.007(0.287)$ & $-0.002(0.288)$ & $0.025(0.083)$ & $0.001(0.076)$ & $-0.000(0.076)$ & $-0.002(0.076)$ \\
\hline & 40 & $0.048(0.267)$ & $-0.000(0.245)$ & $-0.005(0.243)$ & $-0.001(0.245)$ & $0.019(0.069)$ & $0.001(0.064)$ & $0.000(0.064)$ & $-0.001(0.065)$ \\
\hline & 50 & $0.037(0.231)$ & $-0.001(0.216)$ & $-0.004(0.215)$ & $0.001(0.218)$ & $0.015(0.060)$ & $0.001(0.057)$ & $0.000(0.057)$ & $-0.001(0.057)$ \\
\hline \multirow{5}{*}{2.0} & 10 & $0.464(1.238)$ & $-0.064(0.653)$ & $-0.047(1.077)$ & $-0.060(0.698)$ & $0.082(0.190)$ & $0.002(0.147)$ & $-0.002(0.157)$ & $-0.017(0.141)$ \\
\hline & 20 & $0.206(0.668)$ & $0.004(0.515)$ & $-0.036(0.537)$ & $0.002(0.533)$ & $0.039(0.110)$ & $0.002(0.096)$ & $-0.001(0.097)$ & $-0.003(0.095)$ \\
\hline & 30 & $0.127(0.474)$ & $0.004(0.403)$ & $-0.016(0.397)$ & $0.003(0.410)$ & $0.025(0.084)$ & $0.001(0.076)$ & $-0.001(0.076)$ & $-0.002(0.076)$ \\
\hline & 40 & $0.091(0.389)$ & $0.002(0.346)$ & $-0.008(0.342)$ & $0.004(0.347)$ & $0.018(0.069)$ & $-0.000(0.064)$ & $-0.001(0.064)$ & $-0.001(0.064)$ \\
\hline & 50 & $0.071(0.334)$ & $0.002(0.304)$ & $-0.005(0.302)$ & $0.003(0.306)$ & $0.014(0.060)$ & $-0.000(0.057)$ & $-0.001(0.057)$ & $-0.001(0.057)$ \\
\hline
\end{tabular}

Table 2: Estimated bias (root mean-squared error) for $\alpha$ and $\beta,(\beta=1.0)$

\begin{tabular}{|c|c|c|c|c|c|c|c|c|c|}
\hline \multirow[b]{2}{*}{$\alpha$} & \multirow[b]{2}{*}{$n$} & \multicolumn{4}{|c|}{ Estimator of $\alpha$} & \multicolumn{4}{|c|}{ Estimator of $\beta$} \\
\hline & & MLE & $\mathrm{BCE}$ & PBE & FBE & MLE & $\mathrm{BCE}$ & PBE & FBE \\
\hline \multirow{5}{*}{0.5} & 10 & $0.015(0.246)$ & $0.005(0.219)$ & $-0.006(0.209)$ & $0.018(0.211)$ & $0.167(0.381)$ & $0.006(0.296)$ & $-0.030(0.289)$ & $-0.053(0.269)$ \\
\hline & 20 & $0.004(0.153)$ & $0.002(0.147)$ & $-0.000(0.146)$ & $0.005(0.146)$ & $0.073(0.215)$ & $-0.001(0.189)$ & $-0.008(0.188)$ & $-0.012(0.185)$ \\
\hline & 30 & $0.003(0.123)$ & $0.001(0.120)$ & $0.000(0.120)$ & $0.002(0.120)$ & $0.048(0.164)$ & $-0.001(0.150)$ & $-0.004(0.150)$ & $-0.005(0.149)$ \\
\hline & 40 & $0.002(0.106)$ & $0.001(0.104)$ & $0.000(0.104)$ & $0.002(0.104)$ & $0.035(0.138)$ & $-0.000(0.129)$ & $-0.002(0.129)$ & $-0.003(0.128)$ \\
\hline & 50 & $0.001(0.095)$ & $0.000(0.093)$ & $0.000(0.093)$ & $0.001(0.093)$ & $0.029(0.121)$ & $0.000(0.114)$ & $-0.001(0.114)$ & $-0.001(0.114)$ \\
\hline \multirow{5}{*}{1.0} & 10 & $0.107(0.493)$ & $0.004(0.361)$ & $-0.046(0.357)$ & $-0.001(0.364)$ & $0.168(0.386)$ & $0.007(0.300)$ & $-0.028(0.293)$ & $-0.030(0.291)$ \\
\hline & 20 & $0.041(0.270)$ & $0.001(0.240)$ & $-0.008(0.235)$ & $0.000(0.244)$ & $0.078(0.219)$ & $0.004(0.191)$ & $-0.004(0.190)$ & $-0.009(0.188)$ \\
\hline & 30 & $0.027(0.210)$ & $0.002(0.195)$ & $-0.002(0.194)$ & $0.001(0.198)$ & $0.051(0.167)$ & $0.002(0.151)$ & $-0.001(0.151)$ & $-0.004(0.151)$ \\
\hline & 40 & $0.019(0.176)$ & $0.001(0.167)$ & $-0.001(0.166)$ & $0.001(0.170)$ & $0.037(0.138)$ & $0.001(0.129)$ & $-0.000(0.129)$ & $-0.004(0.128)$ \\
\hline & 50 & $0.016(0.157)$ & $0.002(0.150)$ & $0.001(0.150)$ & $0.000(0.152)$ & $0.029(0.120)$ & $0.001(0.113)$ & $-0.000(0.113)$ & $-0.003(0.113)$ \\
\hline \multirow{5}{*}{1.5} & 10 & $0.273(0.881)$ & $-0.012(0.527)$ & $-0.072(0.710)$ & $-0.024(0.540)$ & $0.167(0.388)$ & $0.006(0.302)$ & $-0.020(0.305)$ & $-0.031(0.291)$ \\
\hline & 20 & $0.116(0.456)$ & $0.007(0.374)$ & $-0.018(0.366)$ & $-0.002(0.368)$ & $0.075(0.220)$ & $0.001(0.192)$ & $-0.006(0.191)$ & $-0.008(0.191)$ \\
\hline & 30 & $0.073(0.334)$ & $0.006(0.295)$ & $-0.004(0.290)$ & $-0.002(0.288)$ & $0.049(0.165)$ & $0.001(0.151)$ & $-0.003(0.150)$ & $-0.004(0.151)$ \\
\hline & 40 & $0.051(0.269)$ & $0.002(0.246)$ & $-0.003(0.244)$ & $-0.001(0.245)$ & $0.036(0.139)$ & $0.000(0.129)$ & $-0.001(0.129)$ & $-0.002(0.130)$ \\
\hline & 50 & $0.040(0.233)$ & $0.002(0.217)$ & $-0.001(0.216)$ & $0.001(0.218)$ & $0.029(0.121)$ & $0.000(0.114)$ & $-0.001(0.114)$ & $-0.002(0.115)$ \\
\hline \multirow{5}{*}{2.0} & 10 & $0.470(1.271)$ & $-0.062(0.665)$ & $-0.052(1.065)$ & $-0.060(0.699)$ & $0.163(0.370)$ & $0.002(0.287)$ & $-0.007(0.306)$ & $-0.034(0.282)$ \\
\hline & 20 & $0.205(0.670)$ & $0.003(0.517)$ & $-0.037(0.540)$ & $0.002(0.533)$ & $0.076(0.218)$ & $0.002(0.190)$ & $-0.004(0.191)$ & $-0.006(0.190)$ \\
\hline & 30 & $0.125(0.467)$ & $0.003(0.398)$ & $-0.017(0.390)$ & $0.003(0.410)$ & $0.049(0.165)$ & $0.000(0.150)$ & $-0.003(0.150)$ & $-0.003(0.151)$ \\
\hline & 40 & $0.090(0.380)$ & $0.001(0.338)$ & $-0.009(0.334)$ & $0.004(0.347)$ & $0.035(0.137)$ & $-0.000(0.128)$ & $-0.002(0.128)$ & $-0.003(0.128)$ \\
\hline & 50 & $0.068(0.324)$ & $-0.001(0.296)$ & $-0.007(0.294)$ & $0.003(0.306)$ & $0.027(0.120)$ & $-0.001(0.114)$ & $-0.002(0.114)$ & $-0.002(0.115)$ \\
\hline
\end{tabular}

Table 3: Estimated bias (root mean-squared error) for $\alpha$ and $\beta,(\beta=1.5)$

\begin{tabular}{|c|c|c|c|c|c|c|c|c|c|}
\hline \multirow[b]{2}{*}{$\alpha$} & \multirow[b]{2}{*}{$n$} & \multicolumn{4}{|c|}{ Estimator of $\alpha$} & \multicolumn{4}{|c|}{ Estimator of $\beta$} \\
\hline & & MLE & $\mathrm{BCE}$ & PBE & FBE & MLE & $\mathrm{BCE}$ & PBE & FBE \\
\hline \multirow{5}{*}{0.5} & 10 & $0.015(0.246)$ & $0.005(0.219)$ & $-0.006(0.209)$ & $0.018(0.211)$ & $0.250(0.572)$ & $0.009(0.444)$ & $-0.045(0.436)$ & $-0.079(0.403)$ \\
\hline & 20 & $0.004(0.153)$ & $0.002(0.147)$ & $-0.000(0.146)$ & $0.005(0.146)$ & $0.110(0.323)$ & $-0.001(0.283)$ & $-0.013(0.281)$ & $-0.018(0.278)$ \\
\hline & 30 & $0.003(0.123)$ & $0.001(0.120)$ & $0.000(0.120)$ & $0.002(0.120)$ & $0.071(0.246)$ & $-0.001(0.225)$ & $-0.006(0.225)$ & $-0.008(0.223)$ \\
\hline & 40 & $0.002(0.106)$ & $0.001(0.104)$ & $0.000(0.104)$ & $0.002(0.104)$ & $0.053(0.207)$ & $-0.001(0.193)$ & $-0.003(0.193)$ & $-0.005(0.192)$ \\
\hline & 50 & $0.001(0.095)$ & $0.000(0.093)$ & $0.000(0.093)$ & $0.001(0.093)$ & $0.043(0.181)$ & $0.000(0.171)$ & $-0.001(0.171)$ & $-0.002(0.171)$ \\
\hline \multirow{5}{*}{1.0} & 10 & $0.108(0.504)$ & $0.004(0.366)$ & $-0.045(0.371)$ & $-0.001(0.364)$ & $0.253(0.583)$ & $0.011(0.453)$ & $-0.040(0.446)$ & $-0.045(0.436)$ \\
\hline & 20 & $0.041(0.271)$ & $0.001(0.241)$ & $-0.009(0.235)$ & $0.000(0.244)$ & $0.117(0.328)$ & $0.005(0.285)$ & $-0.006(0.284)$ & $-0.014(0.283)$ \\
\hline & 30 & $0.026(0.210)$ & $0.001(0.195)$ & $-0.003(0.193)$ & $0.001(0.198)$ & $0.076(0.249)$ & $0.003(0.226)$ & $-0.001(0.226)$ & $-0.006(0.226)$ \\
\hline & 40 & $0.019(0.176)$ & $0.001(0.167)$ & $-0.001(0.166)$ & $0.001(0.170)$ & $0.055(0.207)$ & $0.002(0.193)$ & $-0.001(0.193)$ & $-0.006(0.192)$ \\
\hline & 50 & $0.016(0.157)$ & $0.002(0.150)$ & $0.000(0.150)$ & $0.000(0.152)$ & $0.044(0.180)$ & $0.001(0.170)$ & $-0.000(0.170)$ & $-0.005(0.169)$ \\
\hline \multirow{5}{*}{1.5} & 10 & $0.270(0.879)$ & $-0.013(0.525)$ & $-0.077(0.693)$ & $-0.024(0.540)$ & $0.244(0.572)$ & $0.004(0.446)$ & $-0.034(0.452)$ & $-0.047(0.437)$ \\
\hline & 20 & $0.116(0.450)$ & $0.007(0.370)$ & $-0.019(0.359)$ & $-0.002(0.368)$ & $0.115(0.330)$ & $0.004(0.288)$ & $-0.007(0.286)$ & $-0.012(0.287)$ \\
\hline & 30 & $0.075(0.336)$ & $0.007(0.297)$ & $-0.003(0.292)$ & $-0.002(0.288)$ & $0.074(0.249)$ & $0.002(0.227)$ & $-0.003(0.226)$ & $-0.005(0.227)$ \\
\hline & 40 & $0.052(0.270)$ & $0.003(0.247)$ & $-0.002(0.245)$ & $-0.001(0.245)$ & $0.054(0.208)$ & $-0.000(0.194)$ & $-0.003(0.193)$ & $-0.003(0.195)$ \\
\hline & 50 & $0.040(0.234)$ & $0.002(0.218)$ & $-0.001(0.217)$ & $0.001(0.218)$ & $0.043(0.181)$ & $0.001(0.170)$ & $-0.001(0.170)$ & $-0.003(0.172)$ \\
\hline \multirow{5}{*}{2.0} & 10 & $0.460(1.249)$ & $-0.067(0.658)$ & $-0.052(1.078)$ & $-0.060(0.699)$ & $0.246(0.558)$ & $0.006(0.432)$ & $-0.008(0.460)$ & $-0.050(0.424)$ \\
\hline & 20 & $0.205(0.679)$ & $0.003(0.523)$ & $-0.038(0.541)$ & $0.002(0.533)$ & $0.117(0.328)$ & $0.005(0.285)$ & $-0.004(0.286)$ & $-0.009(0.285)$ \\
\hline & 30 & $0.127(0.476)$ & $0.004(0.405)$ & $-0.016(0.399)$ & $0.003(0.410)$ & $0.075(0.250)$ & $0.003(0.227)$ & $-0.002(0.227)$ & $-0.005(0.227)$ \\
\hline & 40 & $0.088(0.379)$ & $0.000(0.337)$ & $-0.010(0.333)$ & $0.004(0.347)$ & $0.055(0.207)$ & $0.001(0.192)$ & $-0.002(0.192)$ & $-0.004(0.192)$ \\
\hline & 50 & $0.069(0.326)$ & $-0.000(0.298)$ & $-0.006(0.296)$ & $0.003(0.306)$ & $0.042(0.180)$ & $-0.000(0.170)$ & $-0.002(0.170)$ & $-0.004(0.172)$ \\
\hline
\end{tabular}


Table 4: Estimated bias (root mean-squared error) for $\alpha$ and $\beta,(\beta=2.0)$

\begin{tabular}{|c|c|c|c|c|c|c|c|c|c|}
\hline \multirow[b]{2}{*}{$\alpha$} & \multirow[b]{2}{*}{$n$} & \multicolumn{4}{|c|}{ Estimator of $\alpha$} & \multicolumn{4}{|c|}{ Estimator of $\beta$} \\
\hline & & MLE & $\mathrm{BCE}$ & PBE & FBE & MLE & $\mathrm{BCE}$ & PBE & FBE \\
\hline \multirow{5}{*}{0.5} & 10 & $0.015(0.246)$ & $0.005(0.219)$ & $-0.007(0.209)$ & $0.018(0.211)$ & $0.333(0.763)$ & $0.011(0.592)$ & $-0.056(0.595)$ & $-0.106(0.537)$ \\
\hline & 20 & $0.004(0.153)$ & $0.002(0.147)$ & $-0.000(0.146)$ & $0.005(0.146)$ & $0.146(0.431)$ & $-0.002(0.377)$ & $-0.017(0.375)$ & $-0.024(0.370)$ \\
\hline & 30 & $0.003(0.123)$ & $0.001(0.120)$ & $0.000(0.120)$ & $0.002(0.120)$ & $0.095(0.329)$ & $-0.001(0.300)$ & $-0.007(0.299)$ & $-0.011(0.298)$ \\
\hline & 40 & $0.002(0.106)$ & $0.001(0.104)$ & $0.000(0.104)$ & $0.002(0.104)$ & $0.070(0.276)$ & $-0.001(0.257)$ & $-0.004(0.257)$ & $-0.006(0.256)$ \\
\hline & 50 & $0.001(0.095)$ & $0.000(0.093)$ & $0.000(0.093)$ & $0.001(0.093)$ & $0.057(0.242)$ & $0.000(0.229)$ & $-0.002(0.228)$ & $-0.003(0.228)$ \\
\hline \multirow{5}{*}{1.0} & 10 & $0.103(0.491)$ & $0.001(0.360)$ & $-0.048(0.357)$ & $-0.001(0.364)$ & $0.349(0.785)$ & $0.025(0.607)$ & $-0.040(0.607)$ & $-0.060(0.582)$ \\
\hline & 20 & $0.042(0.275)$ & $0.001(0.245)$ & $-0.008(0.239)$ & $0.000(0.244)$ & $0.160(0.443)$ & $0.011(0.384)$ & $-0.004(0.382)$ & $-0.018(0.377)$ \\
\hline & 30 & $0.026(0.209)$ & $0.001(0.194)$ & $-0.003(0.193)$ & $0.001(0.198)$ & $0.100(0.331)$ & $0.003(0.301)$ & $-0.003(0.301)$ & $-0.008(0.302)$ \\
\hline & 40 & $0.019(0.178)$ & $0.001(0.169)$ & $-0.001(0.168)$ & $0.001(0.170)$ & $0.075(0.276)$ & $0.003(0.257)$ & $-0.000(0.256)$ & $-0.008(0.257)$ \\
\hline & 50 & $0.015(0.158)$ & $0.001(0.151)$ & $-0.000(0.151)$ & $0.000(0.152)$ & $0.060(0.244)$ & $0.004(0.230)$ & $0.001(0.230)$ & $-0.007(0.225)$ \\
\hline \multirow{5}{*}{1.5} & 10 & $0.287(0.893)$ & $-0.002(0.532)$ & $-0.064(0.705)$ & $-0.024(0.540)$ & $0.346(0.768)$ & $0.022(0.592)$ & $-0.025(0.609)$ & $-0.062(0.582)$ \\
\hline & 20 & $0.110(0.444)$ & $0.003(0.366)$ & $-0.023(0.354)$ & $-0.002(0.368)$ & $0.153(0.437)$ & $0.004(0.381)$ & $-0.010(0.379)$ & $-0.016(0.382)$ \\
\hline & 30 & $0.068(0.325)$ & $0.002(0.288)$ & $-0.008(0.283)$ & $-0.002(0.288)$ & $0.100(0.331)$ & $0.003(0.301)$ & $-0.003(0.301)$ & $-0.007(0.303)$ \\
\hline & 40 & $0.051(0.269)$ & $0.003(0.246)$ & $-0.002(0.244)$ & $-0.001(0.245)$ & $0.074(0.275)$ & $0.003(0.256)$ & $-0.001(0.256)$ & $-0.005(0.260)$ \\
\hline & 50 & $0.040(0.232)$ & $0.002(0.216)$ & $-0.001(0.215)$ & $0.001(0.218)$ & $0.057(0.241)$ & $0.001(0.227)$ & $-0.002(0.227)$ & $-0.004(0.230)$ \\
\hline \multirow{5}{*}{2.0} & 10 & $0.454(1.234)$ & $-0.070(0.656)$ & $-0.055(1.066)$ & $-0.060(0.699)$ & $0.324(0.747)$ & $0.004(0.580)$ & $-0.011(0.624)$ & $-0.067(0.565)$ \\
\hline & 20 & $0.200(0.664)$ & $-0.001(0.514)$ & $-0.041(0.534)$ & $0.002(0.533)$ & $0.148(0.435)$ & $0.000(0.380)$ & $-0.012(0.382)$ & $-0.013(0.380)$ \\
\hline & 30 & $0.121(0.466)$ & $-0.002(0.398)$ & $-0.021(0.391)$ & $0.003(0.410)$ & $0.093(0.329)$ & $-0.003(0.301)$ & $-0.009(0.301)$ & $-0.007(0.302)$ \\
\hline & 40 & $0.090(0.384)$ & $0.002(0.342)$ & $-0.009(0.338)$ & $0.004(0.347)$ & $0.071(0.279)$ & $-0.001(0.260)$ & $-0.004(0.260)$ & $-0.006(0.256)$ \\
\hline & 50 & $0.072(0.334)$ & $0.003(0.304)$ & $-0.003(0.302)$ & $0.003(0.306)$ & $0.057(0.243)$ & $0.001(0.230)$ & $-0.001(0.230)$ & $-0.005(0.229)$ \\
\hline
\end{tabular}

In order to evaluate the overall performance of each of the three different estimators, in terms of the bias and root mean-squared error, we adopted two measures introduced by Cribari-Neto and Vasconcellos (2002), also considered in Lemonte (2011). These measures are the integrated bias squared (IBSQ) and the average root mean-squared error (ARMSE) are calculated for each value of $n$ as follows:

$$
\operatorname{IBSQ}_{(n)}=\sqrt{\frac{1}{16} \sum_{h=1}^{16}\left(r_{h, n}\right)^{2}} \quad \text { and } \quad \operatorname{ARMSE}_{(n)}=\frac{1}{16} \sum_{h=1}^{16} \operatorname{RMSE}_{h, n}
$$

where $r_{h, n}$ and $R M S E_{h, n}$ are the estimated bias and estimated root mean-squared error for the $h$-th scenario, $h=1, \ldots, 16$.

The results showed in Tables 5 and 6 indicate that the three corrections, BCEs, PBEs and FBEs, outperform the MLEs. Also, Tables 5 and 6 show that these measures are very close for the BCEs, PBEs and FBEs.

Table 5: Integrated bias squared norm

\begin{tabular}{ccccc|cccc}
\hline \multirow{4}{*}{$n$} & \multicolumn{4}{c|}{ Estimator for $\alpha$} & \multicolumn{4}{c}{ Estimator for $\beta$} \\
\cline { 2 - 9 } & MLE & BCE & PBE & FBE & MLE & BCE & PBE & FBE \\
\hline 10 & 0.2750 & 0.0333 & 0.0499 & 0.0334 & 0.2296 & 0.0103 & 0.0284 & 0.0521 \\
20 & 0.1185 & 0.0033 & 0.0222 & 0.0027 & 0.1042 & 0.0038 & 0.0079 & 0.0126 \\
30 & 0.0731 & 0.0031 & 0.0095 & 0.0022 & 0.0670 & 0.0020 & 0.0039 & 0.0057 \\
40 & 0.0523 & 0.0016 & 0.0049 & 0.0021 & 0.0496 & 0.0014 & 0.0021 & 0.0044 \\
50 & 0.0408 & 0.0014 & 0.0031 & 0.0016 & 0.0395 & 0.0011 & 0.0012 & 0.0032 \\
\hline
\end{tabular}


Table 6: Average root mean-squared error

\begin{tabular}{ccccc|cccc}
\hline \multirow{4}{*}{$n$} & \multicolumn{4}{c|}{ Estimator for $\alpha$} & \multicolumn{4}{c}{ Estimator for $\beta$} \\
\cline { 2 - 9 } & MLE & BCE & PBE & FBE & MLE & BCE & PBE & FBE \\
\hline 10 & 0.8144 & 0.4723 & 0.6731 & 0.4893 & 0.5235 & 0.4057 & 0.4135 & 0.3881 \\
20 & 0.4331 & 0.3484 & 0.3525 & 0.3536 & 0.2987 & 0.2607 & 0.2598 & 0.2585 \\
30 & 0.3124 & 0.2734 & 0.2692 & 0.2759 & 0.2263 & 0.2063 & 0.2058 & 0.2062 \\
40 & 0.2557 & 0.2322 & 0.2301 & 0.2345 & 0.1892 & 0.1763 & 0.1761 & 0.1762 \\
50 & 0.2215 & 0.2054 & 0.2043 & 0.2079 & 0.1655 & 0.1563 & 0.1562 & 0.1562 \\
\hline
\end{tabular}

Based on the results of this simulation study, it is clear that the second-order bias reduction estimators (BCEs, PBEs and FBEs) are more efficient than the MLEs. Finally, although the three methods are equally efficient, the BCEs and FBEs are computationally easier than the PBEs.

\section{Applications}

In this section we consider the two real data sets used in Mazucheli et al. (2018b) to illustrate the importance of the proposed bias-corrected estimators. For both data sets, Mazucheli et al. (2018b) showed the superiority of the unit-Weibull distribution against 8 competing distributions on the unit interval.

The first data set is from Dumonceaux and Antle (1973) and refers to 20 observations of the maximum flood level (in millions of cubic feet per second) for Susquehanna River at Harrisburg, Pennsylvania. The second data set refers to 48 observations obtained from 12 core samples from petroleum reservoirs that were sampled by 4 cross-sections.

The MLEs and their estimated biases are given in Table 7 for both data sets. Note that, for data set II, the estimated biases of the MLE $\widehat{\alpha}$ using the three corrected estimates are negative.

Table 7: Estimated Biases of MLEs

\begin{tabular}{lccccc}
\hline & \multicolumn{2}{c}{ Data Set I } & & \multicolumn{2}{c}{ Data Set II } \\
\cline { 2 - 3 } \cline { 5 - 6 } Method & $\widehat{\alpha}=1.0248$ & $\widehat{\beta}=3.9036$ & & $\widehat{\alpha}=0.0602$ & $\widehat{\beta}=5.1130$ \\
\hline Cox-Snell & 0.0356 & 0.2693 & & -0.0001 & 0.1469 \\
Parametric bootstrap & 0.0464 & 0.3059 & & -0.0003 & 0.1610 \\
Firth & 0.0340 & 0.3336 & & -0.0010 & 0.1428 \\
\hline
\end{tabular}

Table 8 shows the estimated variance-covariance matrices of various estimators, i.e., the inverse expected information matrix evaluated at these estimators. 
Table 8: Estimated variance-covariance matrices of estimators

\begin{tabular}{|c|c|c|c|c|}
\hline Estimator & \multicolumn{2}{|c|}{ Data Set I } & \multicolumn{2}{|c|}{ Data Set II } \\
\hline MLE & $\left(\begin{array}{r}0.057575 \\
-0.048431\end{array}\right.$ & $\left.\begin{array}{r}-0.048431 \\
0.463182\end{array}\right)$ & $\left(\begin{array}{r}0.000555 \\
-0.012603\end{array}\right.$ & $\left.\begin{array}{r}-0.012603 \\
0.331102\end{array}\right)$ \\
\hline $\mathrm{BCE}$ & $\left(\begin{array}{r}0.054519 \\
-0.047387\end{array}\right.$ & $\left.\begin{array}{r}-0.047387 \\
0.401489\end{array}\right)$ & $\left(\begin{array}{r}0.000557 \\
-0.012255\end{array}\right.$ & $\left.\begin{array}{c}-0.012255 \\
0.31235\end{array}\right)$ \\
\hline PBE & $\left(\begin{array}{r}0.053616 \\
-0.047572\end{array}\right.$ & $\left.\begin{array}{r}-0.047572 \\
0.393434\end{array}\right)$ & $\left(\begin{array}{r}0.000559 \\
-0.012248\end{array}\right.$ & $\left.\begin{array}{r}-0.012248 \\
0.310579\end{array}\right)$ \\
\hline FBE & $\left(\begin{array}{r}0.054647 \\
-0.046455\end{array}\right.$ & $\left.\begin{array}{r}-0.046455 \\
0.387394\end{array}\right)$ & $\left(\begin{array}{r}0.000570 \\
-0.012402\end{array}\right.$ & $\left.\begin{array}{r}-0.012402 \\
0.312864\end{array}\right)$ \\
\hline
\end{tabular}

To investigate the variability of the various estimators, we computed the Bootstrap standard errors, based on 20,000 Bootstrap samples (Efron and Tibshirani 1993). The results are presented in Table 9 along with the parameter estimates for both data sets. Note that, for both data sets, the three corrected estimates have smaller bootstrap standard errors than the corresponding uncorrected estimates. Moreover, the three corrected estimates have almost equal bootstrap standard errors.

Table 9: MLEs, BCEs, PBEs and FBEs (Bootstrap standard-errors).

\begin{tabular}{lccccc}
\hline & \multicolumn{2}{c}{ Data Set I } & & \multicolumn{2}{c}{ Data Set II } \\
\cline { 2 - 3 } \cline { 5 - 6 } Estimator & $\alpha$ & $\beta$ & & $\alpha$ & $\beta$ \\
\hline MLE & $1.0248(0.2810)$ & $3.9036(0.7872)$ & & $0.0602(0.0237)$ & $5.1130(0.6157)$ \\
BCE & $0.9892(0.2730)$ & $3.6343(0.7439)$ & & $0.0603(0.0237)$ & $4.9661(0.5980)$ \\
PBE & $0.9784(0.2700)$ & $3.5977(0.7364)$ & & $0.0605(0.0237)$ & $4.9520(0.5963)$ \\
FBE & $0.9907(0.2713)$ & $3.5699(0.7297)$ & & $0.0612(0.0237)$ & $4.9701(0.5947)$ \\
\hline
\end{tabular}

\section{Concluding remarks}

In Mazucheli et al. (2018b) a new distribution, called unit-Weibull distribution, is proposed for modeling data over the unit interval. It was observed that the maximum likelihood estimates of the parameters of the proposed distribution are biased in small samples. For this distribution, the problem of reducing the biases of the MLEs of the parameters was not addressed in the literature. This problem is important to improve the statistical inference associated with this distribution. Here, we have applied three methodologies widely known in the literature for bias reduction to derive second-order bias corrected MLEs for the parameters of the unit-Weibull distribution. The resulting estimates are called bias-corrected, parametric bootstrap estimates and Firth bias corrected estimates. Our simulation results and two real data applications show that the considered three bias-corrected estimates are preferred, in terms of efficiency, over the classical maximum likelihood estimates.

\section{Acknowledgment}

We are grateful to the editor and anonymous referees for their valuable comments which greatly improved the presentation of the paper. Josmar Mazucheli gratefully acknowledge the partial financial support from the Paraná Research Foundation (FA) - Grant 64/2019. 


\section{References}

Cox DR, Snell EJ (1968). "A General Definition of Residuals." Journal of the Royal Statistical Society, Series B., 30(2), 248-275. doi:10.2307/2984505.

Cribari-Neto F, Vasconcellos KLP (2002). "Nearly Unbiased Maximum Likelihood Estimation for the Beta Distribution." Journal of Statistical Computation and Simulation, 72(2), 107118. doi:10.1080/00949650212144.

Davison AC, Hinkley DV (1997). Bootstrap Methods and Their Applications. Cambridge: Cambridge University Press, New York. doi:10.1017/CB09780511802843.

Doornik JA (2007). Object-Oriented Matrix Programming Using Ox, 3rd ed. London: Timberlake Consultants Press and Oxford. URL www.doornik.com.

Dumonceaux R, Antle CE (1973). "Discrimination between the Log-Normal and the Weibull Distributions." Technometrics, 15(4), 923-926. doi:10.2307/1267401.

Edwards AWF (1992). Likelihood (Expanded Edition). Johns Hopkins University Press, Baltimore. ISBN 9780801844430.

Efron B (1982). The Jackknife, the Bootstrap and Other Resampling Plans. CBMS-NSF Reg. Conf. Ser. Appl. Math. SIAM, Philadelphia, PA. doi:10.1137/1.9781611970319. Lectures given at Bowling Green State Univ., June 1980, URL https://cds.cern.ch/ record/98913.

Efron B, Tibshirani RJ (1993). An Introduction to the Bootstrap, volume 57 of Monographs on Statistics and Applied Probability. Chapman and Hall, New York. ISBN 9780412042317.

Firth D (1993). "Bias Reduction of Maximum Likelihood Estimates." Biometrika, 80(1), 27-38. doi:doi:10.2307/2336755.

Giles DE (2012). "Bias Reduction for the Maximum Likelihood Estimators of the Parameters in the Half-Logistic Distribution." Communication in Statistics - Theory and Methods, 41(2), 212-222. doi:10.1080/03610926.2010.521278.

Giles DE, Feng H, Godwin RT (2013). "On the Bias of the Maximum Likelihood Estimator for the Two-Parameter Lomax Distribution." Communications in Statistics - Theory and Methods, 42(11), 1934-1950. doi:10.1080/03610926.2011.600506.

Lehmann EL (1999). Elements of Large-Sample Theory. Springer-Verlag, New York. ISBN 978-0-387-22729-0.

Lemonte AJ (2011). "Improved Point Estimation for the Kumaraswamy Distribution." Journal of Statistical Computation and Simulation, 81(12), 1971-1982. doi:10.1080/00949655. 2010.511621.

Lemonte AJ, Cribari-Neto F, Vasconcellos KLP (2007). "Improved Statistical Inference for the Two-Parameter Birnbaum-Saunders Distribution." Computational Statistics \& Data Analysis, 51(9), 4656-4681. doi:10.1016/j.csda.2006.08.016.

Ling X, Giles DE (2014). "Bias Reduction for the Maximum Likelihood Estimator of the Parameters of the Generalized Rayleigh Family of Distributions." Communications in Statistics - Theory and Methods, 43(8), 1778-1792. doi:10.1080/03610926.2012.675114.

Mazucheli J, Dey S (2018). "Bias-Corrected Maximum Likelihood Estimation of the Parameters of the Generalized Half-Normal Distribution." Journal of Statistical Computation and Simulation, 88(6), 1027-1038. doi:10.1080/00949655.2017.1413649. 
Mazucheli J, Menezes AFB, Dey S (2018a). "Improved Maximum Likelihood Estimators for the Parameters of the Unit-Gamma Distribution." Communications in Statistics - Theory and Methods, 47(15), 3767-3778. doi:10.1080/03610926.2017.1361993.

Mazucheli J, Menezes AFB, Dey S (2019a). "Bias-Corrected Maximum Likelihood Estimators of the Parameters of the Inverse Weibull Distribution." Communications in Statistics Simulation and Computation, 48(7), 2046-2055. doi:10.1080/03610918.2018.1433838.

Mazucheli J, Menezes AFB, Fernandes LB, de Oliveira RP, Ghitany ME (2019b). "The Unit-Weibull Distribution as an Alternative to the Kumaraswamy Distribution for the Modeling of Quantiles Conditional on Covariates." Journal of Applied Statistics. doi: 10.1080/02664763.2019.1657813. In Press.

Mazucheli J, Menezes AFB, Ghitany ME (2018b). "The Unit-Weibull Distribution and Associated Inference." Journal of Applied Probability and Statistics, 2(13), 1-22. URL http://www.isoss.net.

Mazucheli J, Menezes AFB, Nadarajah S (2017). "mle.tools: An R Package for Maximum Likelihood Bias Correction." The $R$ Journal, 9(2), 268-290. URL https://journal. r-project.org/archive/2017/RJ-2017-055/index.html.

Menezes AFB, Mazucheli J (2018). "Improved Maximum Likelihood Estimators for the Parameters of the Johnson SB Distribution." Communications in Statistics - Simulation and Computation. doi:10.1080/03610918.2018.1498892. In Press.

Millar RB (2011). Maximum Likelihood Estimation and Inference. John Wiley \& Sons, Ltd, United Kingdom. ISBN 978-0-470-09482-2.

Pawitan Y (2001). In All Likelihood: Statistical Modelling and Inference Using Likelihood. Oxford University Press, Oxford. ISBN 9780198507659.

R Core Team (2017). R: A Language and Environment for Statistical Computing. R Foundation for Statistical Computing, Vienna, Austria. URL http://www.R-project.org.

Reath J, Dong J, Wang M (2018). "Improved Parameter Estimation of the Log-Logistic Distribution with Applications." Computational Statistics, 33(1), 339-356. doi:10.1007/ s00180-017-0738-y.

Saha K, Paul S (2005). "Bias-Corrected Maximum Likelihood Estimator of the Negative Binomial Dispersion Parameter." Biometrics, 61(1), 179-185. URL http://www.jstor. org/stable/3695660.

Schwartz J, Giles DE (2016). "Bias-Reduced Maximum Likelihood Estimation of the ZeroInflated Poisson Distribution." Communications in Statistics - Theory and Methods, 45(2), 465-478. doi:10.1080/03610926.2013.824590.

Schwartz J, Godwin RT, Giles DE (2013). "Improved Maximum Likelihood Estimation of the Shape Parameter in the Nakagami distribution." Journal of Statistical Computation and Simulation, 83(3), 434-445. doi:10.1080/00949655.2011.615316.

Wang M, Wang W (2017). "Bias-Corrected Maximum Likelihood Estimation of the Parameters of the Weighted Lindley Distribution." Communications in Statistics - Theory and Methods, 46(1), 530-545. doi : 10.1080/03610918.2014.970696. 


\title{
Affiliation:
}

J. Mazucheli

Department of Statistics

Universidade Estadual de Maringá

Maringá, Paraná, Brasil

E-mail: jmazucheli@gmail.com

\author{
A. F. B Menezes \\ Department of Statistics \\ Universidade Estadual de Campinas \\ Campinas, São Paulo, Brasil \\ E-mail: andrefelipemaringa@gmail.com \\ F. Alqallaf \\ Department of Statistics and Operations Research \\ Faculty of Science \\ Kuwait University, Kuwait \\ E-mail: fatemahku@yahoo.com
}

\author{
M. E. Ghitany \\ Department of Statistics and Operations Research \\ Faculty of Science \\ Kuwait University, Kuwait \\ E-mail: meghitany@yahoo.com
}

\section{Austrian Journal of Statistics}

published by the Austrian Society of Statistics

Volume 50

July 2021 http://www.ajs.or.at/

http://www.osg.or.at/

Submitted: 2019-09-02

Accepted: 2020-01-27 